\title{
Research Challenges in Simulation Aided Design of Complex Multi-agent Systems
}

\author{
Michal Pěchouček and Michal Jakob \\ Agent Technology Center, Department of Cybernetics, \\ Czech Technical University in Prague \\ pechoucek@fel.cvut.cz \\ http://www. agents.cz
}

In today's world, we are increasingly surrounded by and reliant on complex systems and infrastructures. Often, these systems behave far from the optimum or even highly undesirable. Roads in our cities are congested, plane trips frequently delayed, computer networks routinely overrun by worms and electricity grids fail in split-second cascade reactions. Our systems have become massively interwoven and interdependent making both highly positive and negative chain reactions possible in critical systems. The systems that surround us, that provide us with communication, energy resources and support our safety and comfort are increasingly decentralized, interconnected and autonomous, with more and more decisions originating at the level of individual subsystems rather than being imposed top-down. These systems are characterized by large numbers of geographically dispersed active entities with a complex network of mutual interactions and feedbacks, together giving rise to dynamic, non-linear emergent behavior which is very difficult to understand and even more difficult to control.

Creation of mechanisms controlling the operation of above described massively connected autonomous systems cannot be on intuition alone - we need tools and techniques which could provide us with foresight regarding the effect on the control mechanism and policies we want to put in place. Scalable, highfidelity agent-based modeling simulation is the right modeling framework using which such foresight can be obtained. Such simulations will provide experimental computational environment supporting the analysis, design, construction, validation and deployment of multi-agent control systems that are tightly connected with real-world heterogeneous distributed systems. Simulation-aided design of such systems will not only accelerate the development of such system, but will also provide the researchers with a laboratory environment for studying problems and concepts of the future, for which current technology and society is not ready yet.

Before the vision of simulation-aided design can be made a reality realized, however, the following open research challenges need to be addressed and will be discussed during the talk: $(i)$ automated construction, calibration and synchronization of the simulation models, $(i i)$ scalability and fidelity of the simulation, (iii) introduction of mixed-mode simulation and $(i v)$ development and rapid prototyping support. 